\title{
Recommendations for the use of pegylated interferon- $\alpha$ in the treatment of classical myeloproliferative neoplasms
}

Cecily J. Forsyth, ${ }^{1}$ Wai-Hoong Chan, ${ }^{1}$ Andrew P. Grigg ${ }^{2}$ Nathalie C. Cook, ${ }^{3,4}$ Steven W. Lane, ${ }^{5}$ Kate L. Burbury, ${ }^{6}$ Andrew C. Perkins, ${ }^{7}$ and David M. Ross ${ }^{8}$

1. Department of Medicine, Wyong Hospital, Wyong, NSW

2. Department of Clinical Haematology and Olivia Newton John Cancer Research Institute, Austin Hospital, Melbourne, VIC

3. Department of Nutrition and Dietetics, Banyule Community Health, Melbourne, VIC

4. MPN Alliance Australia

5. Department of Haematology, Royal Brisbane and Women's Hospital, and Cancer Program, QIMR Berghofer Medical Research Institute, and School of Medicine, University of Queensland, Brisbane, QLD

6. Department of Haematology, Peter MacCallum Cancer Centre, Melbourne, VIC

7. Department of Clinical Haematology, The Alfred Hospital, Melbourne, VIC

8. Department of Haematology, Royal Adelaide Hospital and Flinders Medical Centre, and Centre for Cancer Biology, SA Pathology and University of South Australia, Adelaide, SA

Abstract word count: 134

Text word count: 2937

\section{Abstract}

The classical myeloproliferative neoplasms (MPN) are uncommon clonal haematopoietic malignancies characterised by excessive production of mature blood cells. Clinically they are associated with thrombosis, haemorrhage, varying degrees of constitutional disturbance, and a risk of progression to myelofibrosis or acute myeloid leukaemia. Many of the disease manifestations may be ameliorated by treatment with interferon- $\alpha$ (IFN) but its use in Australian MPN patients has been limited due to the inconvenience of frequent injections and side effects. The pegylated form of IFN is a long-acting preparation which is better tolerated and its Pharmaceutical Benefits Scheme listing is likely to lead to increased usage.

This is the author manuscript accepted for publication and has undergone full peer review but has not been through the copyediting, typesetting, pagination and proofreading process, which may lead to differences between this version and the Version of Record. Please cite this article as doi: 10.1111/imj.14154

This article is protected by copyright. All rights reserved. 
We review the literature on risks and benefits of IFN treatment for MPNs, suggest criteria for patient selection in each of these diseases, and discuss strategies to manage the side effects of pegylated IFN.

This article is protected by copyright. All rights reserved. 


\section{Introduction}

Philadelphia-negative MPNs comprise a heterogeneous group of blood cancers characterised by the accumulation of mature myeloid cells (granulocytes, erythrocytes, and platelets) and include a spectrum of diseases, from essential thrombocythaemia (ET) and polycythemia vera (PV), to the more advanced and aggressive disease of myelofibrosis which can be either primary (PMF) or secondary to antecedent ET/PV. MPNs result from acquired mutations in haematopoietic stem cells that lead to the activation of tyrosine kinase signalling. The most common genetic mutation, found in more than $95 \%$ of patients with $\mathrm{PV}$, and a majority of patients with $\mathrm{ET}$ or PMF, is a valine to phenylalanine substitution within Janus Kinase 2 (JAK2 V617F) leading to constitutive kinase signalling and cytokineindependent growth. ${ }^{1}$ Other common mutations activate signalling through the thrombopoietin receptor (encoded by the MPL gene), or c-terminal mutations in calreticulin $(C A L R) .{ }^{1}$ These mutations can be detected in the peripheral blood of patients with MPN at the time of diagnosis, and throughout treatment.

Life expectancy in PV (median survival 14 years) and PMF (median survival 6 years), and probably also ET (median survival 20 years) is significantly worse than that of the age- and sex-matched general population., ${ }^{2,3,4}$ Life-threatening complications in MPN include thrombosis, haemorrhage, progressive fibrosis leading to marrow failure, and an increased risk of transformation to acute myeloid leukaemia (AML) which is typically resistant to treatment and dramatically shortens patient survival., 
The treatment of MPN patients in Australia has been limited to anti-platelet agents and cytoreductive drugs (primarily hydroxycarbamide $[\mathrm{HC}]$, formerly 'hydroxyurea' and to a lesser extent anagrelide and busulfan) which largely address thrombosis risk and do not alter the disease course. More recently, ruxolitinib has been approved for MF, but is not funded for patients with low-risk MF or PV/ET.

Standard interferon $\alpha$-2a (IFN) (Roferon- $\mathrm{A}^{\circledR}$ ) has been used for more than three decades to treat MPN and is available on the Pharmaceutical Benefits Scheme (PBS) for MPN patients with "excessive thrombocytosis", but has not been widely used as it requires multiple injections per week and is often poorly tolerated due to adverse effects (AEs). ${ }^{7}$ The coupling of IFN to polyethylene glycol (PEG) inhibits its proteolytic breakdown and clearance, and thereby prolongs its half-life allowing for a decreased frequency of administration and better tolerance and adherence. Recently pegylated interferon $\alpha$-2a (PEG-IFN, Pegasys ${ }^{\circledR}$ ) has been listed on the Australian PBS. This paper reviews the evidence for its activity in MPN, its side-effect profile and makes recommendations regarding its optimal use.

It is unclear whether safety and efficacy differ between PEG-IFN (Roche, Pegasys ${ }^{\circledR}$ ) and $\alpha-2 b$ (Merck, Pegintron ${ }^{\circledR}$ ). Throughout this paper we have treated them as equivalent, although the PBS listing applies only to the former.

\section{How the PBS-listing of PEG-IFN eventuated}

The listing of PEG-IFN (Pegasys ${ }^{\circledR}$ ) on the Australian PBS in 2018 is significant as the first ever consumer-instigated PBS drug listing to occur in Australia. This follows 7 years of advocacy by Nathalie Cook (one of the co-authors of this paper), who was diagnosed with PV in 2010, 
and subsequently became a founding member of the patient advocacy group, MPN Alliance Australia.

The normal pathway for PBS listing follows a submission from the relevant pharmaceutical company of a drug with an approved indication from the Therapeutic Goods Administration (TGA), and requires an extensive dossier outlining safety, efficacy, and cost-effectiveness. High quality data of this type may be impossible to produce for rare diseases. In this case the responsible committee agreed that there was an unmet need for PEG-IFN therapy in MPN and requested that Roche Products lodge an application to the Pharmaceutical Benefits Advisory Committee without a TGA approval for this indication.

The unusual pathway that resulted in the PBS listing of Pegasys ${ }^{\circledR}$ for MPN demonstrates the Australian Government's commitment to rare 'neglected' cancers and coincides with a substantial financial commitment from the Medical Research Future Fund to support rare cancer and disease research, recognising that rare and less common cancers receive a disproportionately low share of cancer research funding despite accounting for over $50 \%$ of cancer deaths. $^{8}$

\section{Rationale for using PEG-IFN in MPNs}

Preclinical models of MPN have been developed using the driver gene mutations, and have been instructive in understanding the way in which patients with MPN can respond to treatments such as IFN or targeted inhibitors of JAK2. ${ }^{9,10}$ IFN is non-leukaemogenic and has anti-proliferative, anti-angiogenic, and differentiation effects on haematopoietic progenitors and may preferentially target the malignant clone. ${ }^{11}$ Clinical trials have shown activity of 
pegylated interferon (PEG-IFN) in PV and ET, with normalisation of blood counts seen in the majority of patients and durable disease control. ${ }^{12}$ Long term treatment with PEG-IFN may induce molecular remission (disappearance of the JAK2 V617F allele) in approximately $15 \%$ of patients. ${ }^{13,14,15}$

\section{Data review and recommendations for PEG-IFN in PV and ET}

Phase 2 clinical trials, involving over 400 PV and ET patients, have shown that PEG-IFN induces objective haematological responses in up to $80 \%$ of patients (including freedom from phlebotomy in $60 \%$ of PV patients), reduces thrombotic complications, and improves pruritus. ${ }^{12,16}$ There is a reduction in the abnormal clone in up to $65 \%$ of patients who have a measurable JAK2 V617F mutation and complete molecular responses (undetectable JAK2 V617F) are reported in up to $24 \%$ after 3 years of therapy. ${ }^{12}$ Similarly, durable complete haematological response (CHR) and a reduction in the mutated CALR allele burden, regardless of the type of CALR mutation, have been noted among ET patients treated with PEG-IFN. ${ }^{17}$ An improvement in bone marrow histopathology after PEG-IFN therapy has been reported in both PV and ET patients in small studies that have included serial bone marrow evaluations. $^{18}$

Both haematological and molecular responses tend to be durable and a small percentage of patients maintain CHR after cessation of the drug with follow-up of at least 2 years. ${ }^{18}$ Disappearance of the malignant clone does not mean cure of the MPN, and relapse can rapidly occur after PEG-IFN discontinuation. Furthermore, mutations in genes other than JAK2 may persist in molecular remission. ${ }^{19}$ Larger, randomised studies are required to 
determine whether molecular responses have any impact on the rate of transformation and survival.

Several phase 3 clinical trials comparing PEG-IFN to HC are ongoing and these trials should help define the role of PEG-IFN in MPN treatment. The MPD-RC 112 randomised trial is comparing PEG-IFN (Pegasys ${ }^{\circledR}$ ) to HC in treatment-naïve patients with high-risk PV/ET. ${ }^{20}$ An interim analysis (12-month data) did not show a difference in the primary endpoint of $\mathrm{CHR}$ between HC and PEG-IFN. Grade 3 AEs were more common in patients receiving PEG-IFN $(16 / 36,44 \%)$ than $\mathrm{HC}(5 / 36,14 \%)$. Within the first 6 months the improvement in symptom burden was greater with PEG-IFN than HC, but with longer duration of therapy this advantage dissipated as patient-reported toxicities of PEG-IFN increased over time. ${ }^{21}$

The MPD-RC 111 trial is evaluating responses to PEG-IFN in patients with high-risk ET/PV refractory to or intolerant of $\mathrm{HC}^{22}$ The interim results of this study reported that PEG-IFN achieved a 12-month overall response rate of $69 \%$ and $60 \%$ in ET and PV, respectively. The presence of a CALR mutation in ET patients was associated with superior CHR rates. PEG-IFN did not appear to decrease quality of life for those patients who are able to tolerate treatment, but the rate of treatment discontinuation was high with only $72 \%$ of patients remaining on therapy for over 12 months.

Recently an ultra-long-acting ropeginterferon- $\alpha-2 b$ (RoPEG-IFN) has been evaluated in clinical trials. ${ }^{23}$ This preparation is administered once every 2 weeks but may be reduced to monthly during long-term maintenance treatment. The phase III CONTI-PV study in PV patients showed that 24 months of treatment with RoPEG-IFN achieved a high CHR rate of 
$70.5 \%$ and a significant reduction in the mutant JAK2 allele burden $(69.6 \%$ of patients achieved a partial molecular response) with a low rate of drug discontinuations $(<10 \%)$.

The recently published revised European Leukemia Net management guidelines recommend either HC or PEG-IFN as first-line cytoreductive therapy for patients with PV or ET at any age. PEG-IFN is also included in their recommendations as second-line drug therapies for both PV and ET patients who are intolerant of, or have an inadequate response to, HC. ${ }^{24}$

\section{Data review and recommendations for IFN in MF}

There is no conclusive evidence that any therapy other than allogeneic transplantation alters the risk of progression or transformation in MF. Ruxolitinib is the best available medical therapy for symptomatic MF patients in the intermediate-2 (INT-2) and high risk cohorts, including those with constitutional symptoms and symptomatic splenomegaly. ${ }^{25}$ Australian PBS criteria permit its use in intermediate-1 risk patients with an unsatisfactory response to other therapies (primarily HC, but PEG-IFN might also be considered in this category).

Several small studies have used IFN, most often PEG-IFN, for the treatment of MF (PMF and secondary MF) and observed both clinical and histological responses. The largest such study was from France, and the outcomes of 62 patients treated with MF in a non-randomised trial were recently updated. ${ }^{26}$ The median age of patients was 67 , and the Dynamic International Prognostic Scoring System (DIPSS) classification was Low/INT-1/INT-2/High in $16 \% / 37 \% / 43 \% / 5 \%$, respectively. The under-representation of high risk patients likely 
reflects previous experience showing poor tolerability and limited efficacy of IFN in patients with advanced disease. Responses in the same study were reported in an earlier publication. ${ }^{27}$ Splenic responses were seen in $47 \%$ of patients and constitutional symptoms resolved in $82 \%$. Thrombocytosis and leukocytosis improved in around $60-80 \%$, whereas thrombocytopenia and leukopenia improved in around $60 \%$ of evaluable patients. Anaemia improved in $72 \%$ of evaluable patients and $39 \%$ of transfusion-dependent patients became transfusion-independent. A $50 \%$ reduction in mutant allele burden was seen in $37 \%$ of JAK2positive patients who had serial quantitative tests performed. In a multivariable model the only significant predictor of partial response (morphologic remission in peripheral blood and resolution of clinical manifestations of disease but without bone marrow histological normalisation) was palpable spleen length (optimal cut-off of $<6 \mathrm{~cm}$ below the costal margin). ${ }^{28}$ The median duration of PEG-IFN treatment was 39 months. A little over half of the patients who discontinued treatment did so due to disease progression. Overall survival was 70 months from diagnosis, which is not dissimilar to that of the historical DIPSS cohort, considering that the study population is dominated by intermediate risk MF. ${ }^{29}$ Seven patients who proceeded to allogeneic stem cell transplantation after discontinuation of PEG-IFN all died, with a high rate of graft-versus-host disease. Patients who received ruxolitinib after PEG-IFN ( $n=15)$ had a median survival of only 22 months, which is shorter than was observed in the ruxolitinib arms of the COMFORT studies. ${ }^{25,30}$ In low risk PMF, where reduction in blood counts and thrombosis risk is the main aim of treatment, the choice of first-line therapy is likely to be between HC and PEG-IFN. As in 
ET/PV the factors that are likely to influence this decision include convenience, toxicity profile, and the perceived importance of molecular responses.

In intermediate risk MF patients the choice is likely to be between PEG-IFN and ruxolitinib. There is no direct comparison of PEG-IFN and ruxolitinib on which to make an evidencebased recommendation. Situations in which PEG-IFN might be preferable include patients with a high risk of infection (e.g. latent hepatitis B virus or Mycobacterial infection), and those for whom improvement of cytopenia is a major goal of treatment. Conversely, the evidence for benefit with ruxolitinib is strongest in patients with higher risk MF, bulky splenomegaly, and a high symptom burden.

\section{Dosing and administration of PEG-IFN}

We recommend a low starting dose of $45 \mu \mathrm{g} /$ week and gradual dose escalation in increments of $45 \mu \mathrm{g} /$ week as tolerated. The 'flu-like side effects (fever, myalgia, chills) which may occur after each injection can be reduced by pre-medication with $1000 \mathrm{mg}$ paracetamol, and by administering the injection at night. Paracetamol should be continued regularly for 1-3 days until the symptoms resolve. 'Flu-like symptoms usually settle with repeated dosing but often recur with each dose increase; hence the dose should only be increased once tolerability at each dose level has been confirmed.

The main goal of treatment is normalisation of elevated blood counts, which is often only achieved after several months of PEG-IFN therapy. Therefore, patients switching from an oral cytoreductive agent to PEG-IFN should ideally continue oral therapy until a CHR is achieved before gradually weaning the oral medication. Blood count control is achieved in 
many patients at a dose of $90 \mu \mathrm{g} /$ week and doses above $180 \mu \mathrm{g} /$ week are usually poorly tolerated. ${ }^{31}$ Once normalisation of blood counts is achieved the dose of PEG-IFN may be tapered to the lowest dose that maintains normal blood counts. Reducing the frequency of injections to fortnightly is achievable for many patients after 1-2 years of therapy.

Despite the improved tolerability of PEG-IFN, toxicity remains a significant issue and approximately $20 \%$ of patients in trials have discontinued PEG-IFN due to AEs, with the majority of discontinuations occurring in the first year of treatment. ${ }^{14,31}$ Table 1 lists the reported haematological and non-haematological $A E$ from a large international cohort of MPN patients treated with PEG-IFN. ${ }^{31}$ The most notable side effects other than 'flu-like effects include fatigue, headaches, alopecia, abnormal liver function tests, and immunemediated phenomena, particularly thyroid dysfunction. ${ }^{31}$ Fatigue is a common adverse event reported in $20-25 \%$ of patients in most series but usually improves with continuing therapy and is only infrequently the major precipitant for treatment discontinuation. Hair loss is usually manifest as thinning, which tends to reach a plateau rather than continuing to worsen. Abnormal liver function tests are usually transient and rarely lead to discontinuation. Autoimmune thyroid dysfunction (hypothyroidism and hyperthyroidism) occurs in up to $5 \%$ of patients on IFN and appears more common in women and possibly also during the first year of therapy. ${ }^{32}$ Thyroid function tests should be performed at baseline and at least annually during treatment, although more frequent monitoring could be considered for female patients during the first year of therapy. No dose reduction is necessary in renal impairment even though approximately $40 \%$ of the total clearance is 
renal. ${ }^{33}$ Patients with renal disease on haemodialysis have been excluded from most studies with PEG-IFN. ${ }^{34}$ Appropriate patient selection, education, and the support of an experienced haematology clinical team can assist in minimising discontinuation due to toxicity.

A history of depression is a relative contraindication to therapy with PEG-IFN. Depression, suicidal ideation, and attempted suicide have been reported during treatment and within six months following discontinuation. ${ }^{35}$ Patients should be evaluated for signs or symptoms of mood disorders and, if depression develops or worsens, PEG-IFN should be discontinued and psychiatric intervention provided, as appropriate.

\section{Monitoring response to treatment}

MPN treatment response criteria have been described by the European Leukemia Net (ELN) however these are predominantly used in the clinical trial setting. ${ }^{28,36}$ Clinically relevant assessments of response would be based on resolution of symptoms and signs, and normalisation of blood counts. Table 3 details the current definitions of response in ET and PV. Effects on marrow fibrosis and histology can only be assessed by a bone marrow biopsy. Outside a clinical trial setting there is no standard approach to follow-up histological assessment. At the discretion of the treating haematologist and the patient, a repeat marrow examination could be considered after several years of haematological response, particularly in patients with MF where successful clinical response correlates with an improvement in bone marrow morphology (reduction in marrow fibrosis, cellularity, and megakaryocyte density). ${ }^{37}$ Measuring molecular response by the allele burden of JAK2 V617F (or other mutations) is not currently standard practice in Australia. Complete 
response from a molecular viewpoint is defined as eradication of the pre-existing abnormality and a partial response as $\geq 50 \%$ decrease in allele burden. ${ }^{28}$ Measurement could be considered after one to two years of PEG-IFN, especially to aid in decisions regarding dose reduction or discontinuation.

\section{PEG-IFN in pregnancy}

Only a minority of MPN cases are diagnosed in women of childbearing age. Pregnancy in patients with ET is associated with an increase in foetal (spontaneous abortion, stillbirth, intrauterine growth retardation) and maternal complications (thrombosis, hemorrhage, preeclampsia). ${ }^{38}$ Most published experience is in ET and expert recommendations for PV are extrapolated from the experience in ET. Current expert advice for patients with low-risk disease (no prior thrombosis or MPN-related pregnancy complications, and platelets $<1500$ x $10^{9} / \mathrm{L}$ ) does not recommend cytoreduction, but includes low dose aspirin and - in PV patients - phlebotomy to maintain haematocrit less than $40 \% .^{38,39}$ In patients with high-risk disease cytoreduction with IFN is recommended. ${ }^{38}$ There has been substantial experience with conventional IFN in pregnancy and there are emerging data with PEG-IFN which is considered safe to use in all trimesters (category B3) as placental transfer appears unlikely. In a series of 10 pregnancies in 8 women with ET treated with PEG-IFN, treatment was started before conception for patients with high-risk disease. ${ }^{40}$ Additional therapy included aspirin and low molecular weight heparin. The median platelet count fell progressively throughout pregnancy from $509 \times 10^{9} / \mathrm{L}$ preconception to $285 \times 10^{9} / \mathrm{L}$ at delivery. Neither major bleeding nor thrombosis was reported and there were no grade 3-4 AEs nor drug 
discontinuation. Nine live births (with no infant malformations) and one miscarriage were reported.

PEG-IFN is considered safe to use during breastfeeding as the drug is excreted into breast milk in very small amounts and is not absorbed from the gastrointestinal lumen. ${ }^{41}$

\section{Conclusion}

The therapeutic armamentarium for MPN in Australia has been expanded by the recent public funding of PEG-IFN and table 2 summarises what we feel are reasonable indications for its use. Despite this, many unmet patient needs remain, including normalisation of lifespan, prevention of haematological progression, and improved quality of life. PEG-IFN may make a modest contribution to addressing these unmet needs, especially in ET/PV. Major advances are likely only with the development of novel therapies with more specific disease-modifying activity. 


\begin{tabular}{|l|c|}
\hline & Number with AE (\% of total) \\
\hline Haematological & $10(8 \%)$ \\
\hline Thrombocytopenia & $7(6 \%)$ \\
\hline Anaemia & $7(6 \%)$ \\
\hline Leukopenia & \\
\hline Non-haematological & $24(20 \%)$ \\
\hline Fatigue & $7(6 \%)$ \\
\hline LFT elevation & $6(5 \%)$ \\
\hline Skin/allergic reaction & $5(4 \%)$ \\
\hline Nausea & $5(4 \%)$ \\
\hline Mood disorder & $3(2.5 \%)$ \\
\hline Headache & $3(2.5 \%)$ \\
\hline Alopecia & $3(2.5 \%)$ \\
\hline Myalgia & $2(2 \%)$ \\
\hline Stomatitis & $1(<1 \%)$ \\
\hline Thyroiditis & $1(<1 \%)$ \\
\hline Cough & \\
\hline & \\
\hline & \\
\hline & \\
\hline
\end{tabular}

Table 1: Adverse events in 118 MPN patients treated with PEG-IFN ${ }^{17}$ 


\section{ET/PV patients for whom PEG-IFN should be considered:}

(patients meeting any of the following criteria)

First-line cytoreductive therapy, particularly in younger patients

Patients requiring cytoreductive therapy who are planning a pregnancy

Second-line therapy for patients with intolerance or resistance to $\mathrm{HC}$

MF patients for whom PEG-IFN should be considered:

(patients meeting all of the following criteria)

Low or intermediate risk disease with proliferative features

Absence of massive splenomegaly ( $\leq 5 \mathrm{~cm}$ below costal margin)

Absence of marked leukopenia or thrombocytopenia

Less severe bone marrow fibrosis (MF 0-2)

This article is protected by copyright. All rights reserved. 
Not planned for allogeneic transplantation in the next 12 months

Table 2: Recommendations for patient selection for PEG-IFN treatment

\begin{tabular}{|c|c|c|}
\hline Response grade & Response in polycythaemia vera & Response in essential thrombocythaemia \\
\hline $\begin{array}{l}\text { Complete } \\
\text { Response } \\
\text { (all criteria met) }\end{array}$ & $\begin{array}{l}\text { 1. Ht }<45 \% \text { without phlebotomy, } \\
\text { 2. Platelet count } \leq 400 \times 10^{9} / \mathrm{L} \text {, } \\
\text { 3. WBC count } \leq 10 \times 10^{9} / \mathrm{L} \text {, } \\
\text { 4. Normal spleen size, AND } \\
\text { 5. No disease related symptoms }{ }^{\dagger}\end{array}$ & $\begin{array}{l}\text { 1. Platelet count } \leq 400 \times 10^{9} / \mathrm{L} \text {, } \\
\text { 2. } \mathrm{WBC} \text { count } \leq 10 \times 10^{9} / \mathrm{L} \text {, } \\
\text { 3. Normal spleen size, AND } \\
\text { 4. No disease related symptoms }{ }^{\dagger}\end{array}$ \\
\hline Partial Response & $\begin{array}{l}\text { 1. } \mathrm{Ht}<45 \% \text { without phlebotomy, } \\
\mathrm{OR} \\
\text { 2. Response in } \geq 3 \text { other above } \\
\text { criteria }\end{array}$ & $\begin{array}{l}\text { 1. Platelet count } \leq 600 \times 10^{9} / \mathrm{L} \text { or } \\
\text { decrease of }>50 \% \text { from baseline }\end{array}$ \\
\hline No Response & Does not satisfy partial response & Does not satisfy partial response \\
\hline
\end{tabular}

Response criteria should be observed for at least 12 weeks.

$\mathrm{Ht}$, haematocrit. WBC, white blood cell

This article is protected by copyright. All rights reserved. 
Table 3. European Leukaemia Net definitions of clinical and haematological responses in PV and $\mathrm{ET}^{28}$ 


\section{References}

1. Nangalia J, Green TR. The evolving genomic landscape of myeloproliferative neoplasms. Hematology Am Soc Hematol Educ Program 2014; 287-296.

2. Tefferi A, Barbui T. Polycythemia vera and essential thrombocythemia: 2017 update on diagnosis, risk-stratification, and management. AmJ Haematol 2017; 92: 94-108.

3. Hultcrantz M, Kristinsson SY, Andersson T, Landgren O, Eloranta S, Derolf A et al. Patterns of survival among patients with myeloproliferative neoplasms diagnosed in Sweden from 1973 to 2008: A population-based study. J Clin Oncol 2012; 30: 2995-3001

4. Tefferi A, Guglielmelli P, Larson DR, Finke C, Wassie EA, Pieri L, et al. Long-term survival and blast transformation in molecularly annotated essential thrombocythaemia, polycythaemia vera, and myelofibrosis. Blood 2014; 124: 2507-13.

5. Caramazza D, Begna K, Gangat N, Vaidya R, Siragusa S, Van Dyke DL et al. Refined cytogenetic-risk categorization for overall and leukemia-free survival in primary myelofibrosis: a single center study of 433 patients. Leukemia 2011; 25: 82.

6. Kennedy JA, Atenafu EG, Messner HA, Craddock KJ, Brandwein JM, Lipton JH et al. Treatment outcomes following leukemic transformation in Philadelphia-negative myeloproliferative neoplasms. Blood 2013; 121: 2725-33.

7. Bellucci S, Harousseau JL, Brice P, Tobelem G. Treatment of essential thrombocythaemia by alpha 2a interferon. Lancet 1988; 2: 960-1.

8. Rare Cancers Australia, Just a Little More Time: Rare Cancers Update Report 2016.

9. Mullally A, Lane SW, Ball B, Megerdichian C, Okabe R, Al-Shahrour F et al. Physiological Jak2V617F expression causes a lethal myeloproliferative neoplasm with differential effects on hematopoietic stem and progenitor cells. Cancer Cell 2010; 17: 584-96.

10. Mullally A, Bruedigam C, Poveromo L, Heidel FH, Purdon A, Vu T et al. Depletion of JAK2V617F myeloproliferative neoplasm-propagating stem cells by interferon- $\alpha$ in a murine model of polycythemia vera. Blood 2013; 121: 3692-702.

This article is protected by copyright. All rights reserved. 
11. Shreenivas A, Mascarenhas J. Emerging drugs for the treatment of Myelofibrosis. Expert Opin Emerg Drugs 2018; 23: 37-49.

12. Kiladjian J-J, Giraudier S, Cassinat B. Interferon-alpha for the therapy of myeloproliferative neoplasms: targeting the malignant clone. Leukemia 2016; 30: 776-781.

13. Kiladjian J-J, Cassinat B, Chevret S, Turlure P, Cambier N, Roussel M et al. Pegylated interferon-alfa-2a induces complete hematologic and molecular responses with low toxicity in polycythemia vera. Blood 2008; 112: 3065-72.

14. Quintás-Cardama A, Abdel-Wahab O, Manshouri T, Kilpivaara O, Cortes J, Roupie A-L, et al. Molecular analysis of patients with polycythemia vera or essential thrombocythemia receiving pegylated interferon $\alpha-2$ a. Blood 2013; 122: 893-901.

15. Them N, Bagienski K, Berg T, Gisslinger B, Schalling M, Chen D et al. Molecular responses and chromosomal aberrations in patients with polycythemia vera treated with peg-proline-interferon alpha-2b. Am J Hematol 2015; 90: 288-94.

16. Masarova L, Patel KP, Newberry KJ, Cortes J, Borthakur G, Konopleva M, et al. Pegylated interferon alfa-2a in patients with essential thrombocythaemia or polycythaemia vera: a post-hoc, median 83 month follow-up of an open-label, phase 2 trial. Lancet Haematol 2017; 4: e165-e75

17. Verger E, Cassinat B, Chauveau A, Dosquet C, Giraudier S, Schlageter M-H, et al. Clinical and molecular response to interferon- $\alpha$ therapy in essential thrombocythemia patients with CALR mutations. Blood 2015; 126: 2585-91

18. Utke Rank C, Weis Bjerrum O, Larsen TS, Kjær L, de Stricker K, Riley CH et al. Minimal residual disease after long-term interferon-alpha2 treatment: a report on hematological, molecular and histomorphological response patterns in 10 patients with essential thrombocythemia and polycythemia vera. Leuk Lymphoma 2015; 18: 1-7.

19. Kiladjian J-J, Giraudier S, Cassinat B. Interferon-alpha for the therapy of myeloproliferative neoplasms: targeting the malignant clone. Leukaemia 2016; 30: 776-781. 
20. Macarenhas J, Prchal J, Rambaldi A, Mesa R, Berenzon D, Yacoub et al. Interim analysis of the myeloproliferative disorders research Consortium (MPD-RC) 112 global phase III trial of frontline pegylated interferon alfa-2a vs hydroxyurea in high risk polycythaemia and essential thrombocythaemia. Blood 2016 128: 479.

21. Mesa RA, Hoffman R, Kosiorek HE, Prchal JT, Harrison CN, McMullin MF, et al. Impact on MPN symptoms and quality of life of front line pegylated interferon alfa-2a Vs. hydroxyurea in high risk polycythemia vera and essential thrombocythemia: interim analysis results of Myeloproliferative Disorders Research Consortium (MPD-RC) 112 global phase III trial. Blood 2016; 128: 4271.

22. Yacoub A, Mascarenhas J, Kosiorek HE, Prchal JT, Berenzon D, Baer MR, et al. Single-arm salvage therapy with pegylated interferon alfa-2a for patients with high-risk polycythemia vera or high-risk essential thrombocythemia who are either hydroxyurea-resistant or intolerant: final results of the Myeloproliferative Disorders-Research Consortium (MPD-RC) protocol 111 global phase II trial. Blood 2017; 130: 321.

23. Gisslinger H, Klade C, Georgiev P, Krochmalczyk D, Gercheva L, Egyed M, et al. Ropeginterferon Alfa-2b induces high rates of clinical, hematological and molecular responses in polycythemia vera: two-year results from the first prospective randomized controlled trial. Blood 2017; 130: 320.

24. Barbui T, Tefferi A, Vannucchi AM, Passamonit F, Silver RT, Hoffman R, et al. Philadelphia chromosome-negative classical myeloproliferative neoplasms:revised management recommendations from European LeukemiaNet. Leukemia 2018; 32: 1057-10.

25. Harrison CN, Vannucchi AM, Kiladjian J-F, Al-Ali, HK, Gisslinger H, Knoops L et al. Longterm findings from COMFORT-II, a phase 3 study of ruxolitinib vs best available therapy for myelofibrosis. Leukaemia 2016; 30:1701-1707.

26. Ianotto J, Chauveau A, Boyer-Perrard F, Gyan E, Laribi K, Cony-Makhoul P et al. Benefits and pitfalls of Pegylated Interferon-A2a therapy in patients with myeloproliferative 
neoplasm-associated myelofibrosis: A French Intergroup of Myeloproliferative Neoplasms (FIM) study. Haematologica 2018; 103: 438-446.

27. Ianotto JC, Boyer-Perrard F, Gyan E, Laribi K, Cony-Makhoul P, Demory JL, et al. Efficacy and safety of pegylated-interferon $\alpha-2 a$ in myelofibrosis: a study by the FIM and GEM French cooperative groups. Br J Haematol 2013; 162: 783-91.

28. Tefferi A, Cervantes F, Mesa F, Passamonti F, Verstovsek S, Vannucchi A, et al. Revised response criteria for myelofibrosis: International Working Group-Myeloproliferative Neoplasms Research and Treatment (IWG-MRT) and European LeukemiaNet (ELN) consensus report. Blood 2013; 8: 1395-1398.

29. Passamonti F, Cervantes F, Vannucchi AM, Morra E, Rumi E, Pereira A et al. A dynamic prognostic model to predict survival in primary myelofibrosis: a study by the IWG-MRT (International Working Group for Myeloproliferative Neoplasms Research and Treatment). Blood 2009; 115: 1703-8.

30. Verstovsek S, Mesa RA, Gotlib J, Gupta V, DiPersio JF, Catalano JV et al. Long-term treatment with ruxolitinib for patients with myelofibrosis: 5-year update from the randomized, double-blind, placebo-controlled, phase 3 COMFORT-I trial. J Hematol Oncol 2017; 10: 55.

31. Gowin K, Thapaliya P, Samuelson, Harrison C, Radia D, Andreasson B et al. Experience with pegylated interferon alfa-2a in advanced myeloproliferative neoplasms in an international cohort of 118 patients. Haematologica 2012; 97: 1570-1573.

32. Paulsrud C, Hasselbalch HC, Bjerrum OW. Autoimmune thyroid disease in patients with Philadelphia-negative chronic myeloproliferative neoplasms treated with interferon-alpha. $J$ Blood Res Hematol Dis 2016; 1: 1-4.

33. Gupta Sk, Pittenger AL, Swan SK, Marbury TC, Tobillo E Batra V et al. Single-dose pharmacokinetics and safety of pegylated interferon- $\alpha 2 b$ in patients with chronic renal dysfunction. J Clin Pharmacol 2002; 42:1109-1115.

This article is protected by copyright. All rights reserved. 
34. Quintas-Cardama A, Kantarjian H, Manshouri T, Luthra R, Estrov Z, Pierce S et al. J Clin Oncol 2009; 32: 5418-5424.

35. Lotrich F, Rabinovitz M, Gironda P. Depression following pegylated interferon-alpha: characteristics and vulnerability. J Psychosom Res 2007; 63:131-135.

36. Barbui T, Barosi G, Birgegard G, Cervantes F, Finazzi G, Griesshammer M, et al. Philadelphia-negative classical myeloproliferative neoplasms: critical concepts and management recommendations from European LeukemiaNet. J Clin Oncol 2011; 29: 761.

37. Pizzi M, Silver RT, Barel A, Orazi A. Recombinant interferon- $\alpha$ in myelofibrosis reduces bone marrow fibrosis, improves its morphology and is associated with clinical response. Mod Pathol 2015; 28: 1315-1323.

38. Tefferi A, Passamonti F. Essential thrombocythemia and pregnancy: Observations from recent studies and management recommendations. Am J Hematol 2009; 84: 629-630.

39. Vannucchi A. How I treat polycythaemia vera. Blood 2014; 124: 3212-3220.

40. Beauverd Y, Radia D, Cargo C, Knapper S, Drummond M, Pillai A, et al. Pegylated interferon alpha-2a for essential thrombocythemia during pregnancy: outcome and safety. A case series. Haematologica 2016; 101: e182-e4.

41. Kumar AR, Hale TW, Mock RE. Transfer of interferon alfa into human breast milk. J Hum Lact 2000; 16: 226-228.

This article is protected by copyright. All rights reserved. 


\title{
Recommendations for the use of pegylated interferon- $\alpha$ in the treatment of classical myeloproliferative neoplasms.
}

\begin{abstract}
The classical myeloproliferative neoplasms (MPN) are uncommon clonal haematopoietic malignancies characterised by excessive production of mature blood cells. Clinically they are associated with thrombosis, haemorrhage, varying degrees of constitutional disturbance, and a risk of progression to myelofibrosis or acute myeloid leukaemia. Many of the disease manifestations may be ameliorated by treatment with interferon- $\alpha$ (IFN) but its use in Australian MPN patients has been limited due to the inconvenience of frequent injections and side effects. The pegylated form of IFN is a long-acting and better tolerated preparation and its Pharmaceutical Benefits Scheme listing is likely to lead to increased usage. We review the literature on risks and benefits of IFN treatment for MPNs, suggest criteria for patient selection in each of these diseases, and discuss strategies to manage the side effects of pegylated IFN.
\end{abstract}

This article is protected by copyright. All rights reserved. 


\section{Recommendations for the use of pegylated interferon- $\alpha$ in the treatment of classical myeloproliferative neoplasms}

Cecily J. Forsyth, ${ }^{1}$ Wai-Hoong Chan, ${ }^{1}$ Andrew P. Grigg ${ }^{2}$ Nathalie C. Cook, ${ }^{3,4}$ Steven W. Lane, ${ }^{5}$ Kate L. Burbury, ${ }^{6}$ Andrew C. Perkins, ${ }^{7}$ and David M. Ross ${ }^{8}$

1. Department of Medicine, Wyong Hospital, Wyong, NSW

2. Department of Clinical Haematology and Olivia Newton John Cancer Research Institute, Austin Hospital, Melbourne, VIC

3. Department of Nutrition and Dietetics, Banyule Community Health, Melbourne, VIC

4. MPN Alliance Australia

5. Department of Haematology, Royal Brisbane and Women's Hospital, and Cancer Program, QIMR Berghofer Medical Research Institute, and School of Medicine, University of Queensland, Brisbane, QLD

6. Department of Haematology, Peter MacCallum Cancer Centre, Melbourne, VIC

7. Department of Clinical Haematology, The Alfred Hospital, Melbourne, VIC

8. Department of Haematology, Royal Adelaide Hospital and Flinders Medical Centre, and Centre for Cancer Biology, SA Pathology and University of South Australia, Adelaide, SA

Abstract word count: 134

Text word count: 2937 


\section{University Library}

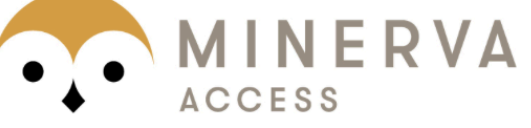

A gateway to Melbourne's research publications

Minerva Access is the Institutional Repository of The University of Melbourne

Author/s:

Forsyth, CJ;Chan, W-H;Grigg, AP;Cook, NC;Lane, SW;Burbury, KL;Perkins, AC;Ross, DM

Title:

Recommendations for the use of pegylated interferon-alpha in the treatment of classical myeloproliferative neoplasms

Date:

2019-08-01

\section{Citation:}

Forsyth, C. J., Chan, W. -H., Grigg, A. P., Cook, N. C., Lane, S. W., Burbury, K. L., Perkins, A. C. \& Ross, D. M. (2019). Recommendations for the use of pegylated interferon-alpha in the treatment of classical myeloproliferative neoplasms. INTERNAL MEDICINE JOURNAL, 49 (8), pp.948-954. https://doi.org/10.1111/imj.14154.

Persistent Link:

http://hdl.handle.net/11343/286227 